\title{
ROLE OF MULTIDETECTOR COMPUTED TOMOGRAPHY IN BILIARY OBSTRUCTION.
}

\author{
Ahmed Mohamed Samy, Hazim Ibrahim Tantawy, Hamed Abdalhakim Gobran, Ahmed \\ Mohamed Alsowey, \\ Radiodiagnosis Departments, Faculty of Medicine, Zagazig, Egypt
}

Corresponding author:

Ahmed Mohamed Samy ahmed.mms@zu.edu.eg

\begin{abstract}
Background: Obstructive Jaundice is a common surgical problem, MultiDetector Computed Tomography in diagnosis of biliary tract obstruction has a vital role as post processing techniques can be utilized to identify variant biliary anatomy to guide preoperative biliary surgery planning, determine level and cause of obstruction, early tumor detection and staging of biliary cancer are the key factors for possible cure by surgical resection, our aim is to evaluate the role of MDCT in diagnosing level and cause of biliary obstruction.

Patients and Methods: Sixty nine patients with clinically suspected biliary obstruction were encountered in the study and subjected to clinical assessment including full history taking, clinical examination and imaging assessment by MDCT, our findings were correlated to standard reference examinations including: Operative/ERCP/Biopsy and histopathology findings.
\end{abstract}

Results: Our results showed that the most affected age group was group between 60: $<70$ years old, males were affected more than females. The most common clinical presentation in our patients was yellowish discoloration of the skin and sclera followed by biliary colic. Right hypochondrial tenderness was the main clinical finding on clinical examination in our study. Our patients were categorized according to the cause of biliary obstruction into seven groups: Malignant stricture group 52.2\% (36patients), Calcular group $24.6 \%$ (17patients), Iatrogenic group 5.8\% (4patients), Porta hepatis lesions group $5.8 \%$ (4patients), Benign stricture group $4.3 \%$ (3patients), Inflammatory group $4.3 \%$ (3patients), Congenital group $1.4 \%$ (1 patient).

Conclusions: MultiDetector computed tomography provides a modality with high diagnostic performance for differentiating benign from malignant lesions and in detecting biliary calculi. Keywords: Multidetector computed tomography; Biliary obstruction; Obstructive jaundice.

\section{INTRODUCTION}

$\mathrm{O}$ bstructive jaundice is a very common surgical problem that happens whenever there is blockage to the passages of conjugated bilirubin from the liver cells to the intestine. Causes of biliary obstruction can be divided into benign or malignant causes ${ }^{(\mathbf{1})}$.

Traditionally, US is the initial imaging technique as a non- invasive cost effective modality for evaluating the biliary obstruction but of lower sensitivity and specificity with limited value in the evaluation of the peripheral intrahepatic ductal lesions and extra hepatic ${ }^{(2)}$.

MRCP is considered the most reliable non-invasive technique that produces high contrast and high-resolution images of the biliary tree and allows evaluation of the solid organs (3). However, contraindications including patients with cardiac pacemakers, cerebral aneurysm clips or claustrophobia, those who cannot endure the long examination, limit added to that high cost and not readily available ${ }^{(4)}$.

Conventional CT or MRI results in high spatial resolution of the biliary tract, but do not provide adequate anatomic information of the bile and the pancreatic ducts ${ }^{(5)}$. For this reason, direct cholangiography such as PTC or ERCP are still used as they are gold standard techniques because they allow both direct visualization of the biliary tree and therapeutic intervention, but they are invasive 
Ahmed et al....

procedures \& carry associated risks that range in severity from minor to life-threatening ${ }^{(6,7)}$.

Recently in the last decade MDCT has led to acquisition of true isotropic voxels that can be post processed to yield images in any plane of the same resolution as the original axially acquired images. Coupled with rapid MDCT imaging during peak target organ enhancement has led to a variety of means to review imaging information beyond that of the axial perspective ${ }^{(7,8)}$. The combined use of MPR and MinIP techniques significantly improves the visualization of the biliary ducts and their site of confluence compared with those obtained by axial CT, also allows displaying of the biliary duct anatomy in various planes. Moreover, MinIP technique enables us to depict the small biliary duct and the pancreatic duct more clearly, MDCT has a vital role as Post processing techniques can be utilized to identify variant biliary anatomy to guide preoperative planning of biliary related surgery ${ }^{(9)}$. Also to determine the level and the cause of biliary obstruction, early tumor detection and staging of the biliary cancer are the key factors for a possible cure by surgical resection, so radiologists helps in improving the patient prognosis by accurate and early diagnosis leading to appropriate therapeutic management for $\mathrm{OJ}^{(\mathbf{1 0 )} \&(\mathbf{1 1})}$.

Aim of the study is to evaluate the role of MDCT in diagnosing the level and cause of biliary obstruction.

\section{PATIENTS AND METHODS}

This was a prospective diagnostic study in concordance with STARD guidelines conducted over a period of six months from May 2018 to October 2018 in Radio diagnosis Department, Zagazig university hospitals. The Data were collected from 69 patients (39 males and 30 females) with majority of cases belonged to the 5th to 6th decade age with clinically supposed OJ. Written consent was obtained from all patients included in our study except for a young patient was obtained from her parents. IRB, Zagazig faculty of medicine has given approval to our study. No conflict of interest.

\section{Patient inclusion criteria:}

1. Any age.

2. Both sexes.

\section{Zagazig University Medical Journals}

3. Cases with clinically suspected biliary obstruction.

\section{Patient exclusion criteria:}

1. All the cases of non-obstructive jaundice.

2. Cases contraindicated for contrast enhanced computed tomography scanning as: Thyroid disease, phaeochromocytoma and Contrast hypersensitivity.

3. Patients with raised renal functions that is not on dialysis.

4. Pregnant females (mainly in the first trimester).

5 . Morbid obesity (>150 kg).

\section{All patients were subjected to the following:}

a. Full history taking:

-Patient personal information.

-Patient complaint.

-Previous medical history.

-History of current condition.

b. Full clinical examination:

This examination is done by internal medicine or general surgery doctors before transfer to our department.

c. Imaging comprising:

Cases will subject to

1) Abdominal US.

2) MDCT of the abdomen.

All cases were scanned by PHILIPS INGENUITY CORE 128.

Scanning parameters were $350 \mathrm{mAs}, 120$ $\mathrm{kVp}$, reconstruction interval of $5 \mathrm{~mm}$, section collimation of 2.5-5 $\mathrm{mm}$ and table speed 7.5$10 \mathrm{~mm}$ per rotation within one breath hold attainment of 8-10 seconds.

Contrast administration by automatic power injector (model mark V: medrad, Indianola, PA)

\section{Patient preparation:}

Fasting for 6 hours. Vigorous oral hydration 2 hours earlier examination. Oral contrast media may be used if needed (iodinated water soluble, $5 \mathrm{~mL} / \mathrm{kg}$ ), Mixed with $500 \mathrm{ml}$ of water and taken $30 \mathrm{~min}$ prior to examination.

Intravenous catheter introduced through antecubital vein.

Creatinine clearance must be $>30 \mathrm{~mL} / \mathrm{min}$, GFR $>45 \mathrm{~mL} / \mathrm{min} / 1.73 \mathrm{~m}^{2}$ or Serum Creatinine level $<1.5 \mathrm{mg} / \mathrm{dl}$.

\section{CT scan Protocol:}

1) Non-contrast phase: 


\section{Ahmed et al....}

Baseline for deciding whether an identifiable lesion enhance, help identifying biliary stones or pancreatic calcification ${ }^{(12)}$.

2) Contrast enhanced phases:

It comprises porto-venous, arterial and late phases. Administration of a nonionic contrast agent (300 $\mathrm{mg}$ of iodine per $\mathrm{mL}$ ) was done intravenously (2 $\mathrm{ml} / \mathrm{kg})$, through the intravenous catheter contrast material was injected at a rate of $3-5 \mathrm{ml} / \mathrm{s}$ for 30 seconds via an programmed power injector, at that time the helical acquisition began $(8 \mathrm{sec})$ after a threshold level of (140HU) was extended in the abdominal aorta then the arterial phase attained (20-30sec) after injection. The portovenous phase developed $(50-60 \mathrm{sec})$ after the injection, finally the delayed phase assimilated (5-10 min) after injection.

\section{Postprocessing Technique:}

The 3D reestablishment with thin planar slicing $(1 \mathrm{~mm})$ and MPR were achieved in coronal and sagittal planes for well description of biliary tract wall and intraluminal lesions. The current introduction

\section{Zagazig University Medical Journals}

of MDCT affords distinctive capabilities that are valued particularly in narrow collimation, hepatic volume achievements, combining short scan periods and the capability to gain multiphase data ${ }^{(\mathbf{1 3})}$.

\section{Standard reference examinations:}

CT findings will be interrelated according to the origin of the obstruction with:

1. Operative/ERCP or PTC in benign stricture, malignancy and calculus.

2. Biopsy and histopathology in malignant masses.

\section{Statistical analysis:}

Through the comparison of MDCT diagnosis with the final diagnosis acquired from standard references inspections, the diagnostic precision of MDCT cholangiography in assessment of reasons of biliary obstruction will be accessed in terms of ACC, SP, SN, PPV and NPV, by tabulating and statistically analyzing collected data, using SPSS 22.0 for windows (SPSS Inc., Chicago, IL, USA) \& MedCalc 13 for windows (MedCalc Software bvba, Ostend, Belgium).

\section{CASE 1}

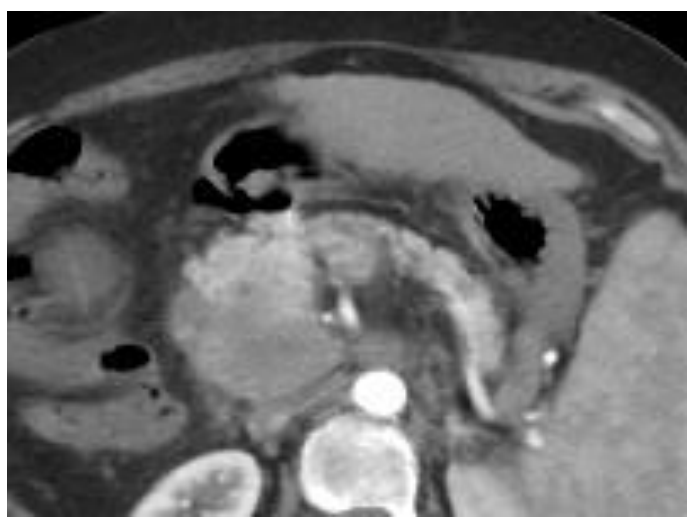

Table 1 Demographic data of the studied cross-section (N=69).

\begin{tabular}{|c|c|c|c|c|c|c|}
\hline \multirow{2}{*}{ Age(years) } & \multicolumn{2}{|c|}{ Male(N=39) } & \multicolumn{2}{c|}{ Female(N=30) } & \multicolumn{2}{c|}{ Total } \\
\cline { 2 - 7 } & No. & $\%$ & No. & $\%$ & No. & $\%$ \\
\hline $0:<10$ & 0 & 0 & 0 & 0 & 0 & 0 \\
\hline $10:<20$ & 0 & 0 & 1 & 1.4 & 1 & 1.4 \\
\hline $20:<30$ & 3 & 4.3 & 4 & 5.8 & 7 & 10.1 \\
\hline $30:<40$ & 2 & 2.9 & 2 & 2.9 & 4 & 5.8 \\
\hline $40:<50$ & 4 & 5.8 & 3 & 4.3 & 7 & 10.1 \\
\hline $50:<60$ & 12 & 17.4 & 3 & 4.3 & 15 & 21.7 \\
\hline $60:<70$ & 14 & 20.3 & 11 & 16 & 25 & 36.3 \\
\hline $70:<80$ & 2 & 2.9 & 5 & 7.2 & 7 & 10.1 \\
\hline $80:<90$ & 2 & 2.9 & 1 & 1.4 & 3 & 4.3 \\
\hline Total & 39 & 56.5 & 30 & 43.5 & 69 & 100 \\
\hline
\end{tabular}


Table 2 MDCT findings regarding degree of biliary dilatation and level of biliary obstruction among the studied cross-section $(\mathrm{N}=69)$.

\begin{tabular}{|l|c|c|}
\hline \multirow{2}{*}{ MDCT findings } & \multicolumn{2}{|c|}{ The studied cross-section(N=69) } \\
\cline { 2 - 3 } & No. & \\
\hline Degree of biliary dilatation & & 58.0 \\
\hline Mild & 40 & 33.3 \\
\hline Moderate & 23 & 8.7 \\
\hline Severe & 6 & 100 \\
\hline Total & 69 & 17.4 \\
\hline Level of biliary obstruction & & 20.3 \\
\hline Intrahepatic & 12 & 62.3 \\
\hline Hilar & 14 & 100 \\
\hline Distal & 43 & \\
\hline Total & 69 & \\
\hline
\end{tabular}

Table 3 Cause of obstruction in MDCT and Gold standard in the studied cross-section (N=69).

\begin{tabular}{|l|c|c|c|c|}
\hline \multirow{2}{*}{ Cause of obstruction } & \multicolumn{4}{|c|}{ The studied cross-section(N=69) } \\
\cline { 2 - 5 } & \multicolumn{2}{|c|}{ MDCT } & \multicolumn{2}{c|}{ Gold standard } \\
\cline { 2 - 5 } & No. & $\%$ & No. & $\%$ \\
\hline Malignant & 41 & 59.4 & 39 & 56.5 \\
\hline Benign & 28 & 40.6 & 30 & 43.5 \\
\hline Total & 69 & 100 & 69 & 100 \\
\hline
\end{tabular}

Table 4 MDCT and Gold standard method diagnosis of the studied cross-section (N=69).

\begin{tabular}{|l|c|c|c|c|}
\hline \multirow{2}{*}{ Diagnosis } & \multicolumn{2}{|c|}{ MDCT } & \multicolumn{2}{c|}{ Gold standard } \\
\cline { 2 - 5 } & No. & $\%$ & No. & $\%$ \\
\hline Malignant stricture & 36 & 52.2 & 36 & 52.2 \\
\hline Calcular & 17 & 24.6 & 17 & 24.6 \\
\hline Iatrogenic & 4 & 5.8 & 4 & 5.8 \\
\hline Porta Hepatis lesions & 4 & 5.8 & 3 & 4.3 \\
\hline Benign stricture & 3 & 4.3 & 5 & 7.2 \\
\hline Inflammatory & 3 & 4.3 & 3 & 4.3 \\
\hline Congenital & 1 & 1.4 & 1 & 1.4 \\
\hline Negative & 1 & 1.4 & - & - \\
\hline Total & 69 & 100 & 69 & 100 \\
\hline
\end{tabular}

Table 5 Type of malignant stricture in MDCT and gold standard method.

\begin{tabular}{|l|c|c|c|c|}
\hline \multirow{2}{*}{ Type of malignant stricture } & \multicolumn{2}{|c|}{ MDCT } & \multicolumn{2}{c|}{ Gold standard method } \\
\cline { 2 - 5 } & No. & $\%$ & No. & $\%$ \\
\hline No malignant stricture & 33 & 47.8 & 33 & 47.8 \\
\hline Cholangiocarcinoma & 14 & 20.3 & 15 & 21.7 \\
\hline Pancreatic carcinoma & 14 & 20.3 & 14 & 20.3 \\
\hline Periampullary carcinoma & 3 & 4.3 & 3 & 4.3 \\
\hline Hepatic focal lesions & 3 & 4.3 & 2 & 2.9 \\
\hline GB carcinoma & 1 & 1.4 & 1 & 1.4 \\
\hline Gastric carcinoma & 1 & 1.4 & 1 & 1.4 \\
\hline Total & 69 & 100 & 69 & 100 \\
\hline
\end{tabular}


Table (6): Diagnostic performance of MDCT.

\begin{tabular}{|l|c|c|c|c|c|}
\hline \multicolumn{1}{|c|}{ Cause } & SN (\%) & SP (\%) & PPV (\%) & NPV (\%) & Acc (\%) \\
\hline Intrahepatic & 100 & 100 & 100 & 100 & 100 \\
\hline Hilar & 100 & 100 & 100 & 100 & 100 \\
\hline Distal & 100 & 100 & 100 & 100 & 100 \\
\hline Malignant cause & 100 & 90.32 & 92.68 & 100 & 95.65 \\
\hline Calcular & 94.12 & 98.08 & 94.12 & 98.08 & 97.10 \\
\hline Benign stricture & 40 & 98.44 & 66.67 & 95.45 & 94.20 \\
\hline Malignant stricture & 94.44 & 93.94 & 94.44 & 93.94 & 94.20 \\
\hline Iatrogenic & 100 & 100 & 100 & 100 & 100 \\
\hline Inflammatory & 100 & 100 & 100 & 100 & 100 \\
\hline Porta-hepatis lesions & 100 & 98.48 & 75 & 100 & 98.55 \\
\hline Congenital & 100 & 100 & 100 & 100 & 100 \\
\hline
\end{tabular}

\section{RESULTS}

Study included 69 patients 30 males and 30 females their ages ranged from 13:82 years, male mean age is 55.6 years and female is 54.2 years making the overall mean age 55 years as shown in table (1)

Biliary dilatation of mild degree was the most observed finding and distal obstruction was the most observed level as shown in table (2).

Regarding the nature of cause that lead to biliary obstruction, malignant causes were more predominant than benign causes as shown in table (3).

Our patients were categorized according to cause of the biliary obstruction into Malignant stricture, Calcular, Iatrogenic, Porta hepatis lesions, Benign stricture, Inflammatory, Congenital and Negative. Most frequent group was malignant stricture and the least frequent group was congenital as shown in table (4).

Malignant stricture findings were further divided to cholangiocarcinoma, pancreatic carcinoma, Periampullary carcinoma, Hepatic focal lesions, GB and Gastric carcinoma as shown in table (5).

Diagnostic performance of MDCT was measured by calculating: SN, SP, PPV, NPV \& NPV for detection of level of biliary obstruction and the cause of biliary obstruction. Regarding the level of obstruction there was high diagnostic performance also in detecting the cause of obstruction it was highest in Iatrogenic, Inflammatory \& Congenital causes and lowest diagnostic performance in detection of
Benign stricture as shown in table (6).

Cases

Case 1: Male patient 55 years old presented by jaundice, abdominal pain and anorexia. (A): Axial oblique reformatted post-contrast (arterial phase) image showing fairly defined pancreatic head mass poorly enhancing.

(B): Coronal oblique MinIP reformatted postcontrast (portal venous phase) image showed moderately dilated CBD till its distal end where the mass encroaches upon it.

DIAGNOSIS: Malignant OJ due to pancreatic head carcinoma with moderate intra- and extra-hepatic biliary dilatation.

Case 2: Male patient 60 years old presented by weight loss and fatty dyspepsia.

(A) Axial contrast (arterial phase) images showing two hepatic focal showing heterogeneous arterial enhancement with pathological circulation within the largest mass. (B): Sagittal oblique reformatted postcontrast (portal venous phase) images showing largest one compressing confluence of CHD exerting mild intra-hepatic biliary radicals dilatation, confined mainly to left side.

DIAGNOSIS: Malignant OJ caused by Multi-focal HCCs exerting mild intra-hepatic biliary radicals dilatation.

Case 3: Female patient 48 years old presented by right hypochondrial pain and attacks of jaundice.

(A): Axial (portal venous phase) image that showed partially contracted GB over dense stones with surrounding minimal pericholecystic free fluid associated with two 


\section{Ahmed et al....}

impacted cystic duct stones exerting extrinsic minimal intrahepatic biliary dilatation and normal caliber CBD.

(B): coronal oblique reformatted post-contrast (portal venous phase) image showed the cystic duct impacted stones and the GB in one plane.

DIAGNOSIS: Calcular OJ due to Mirizzi syndrome due to two impacted cystic duct stones exerting minimal extrinsic intrahepatic biliary dilatation with Calcular cholecystitis.

\section{DISCUSSION}

OJ may lead to mutual surgical problem that can cause life threatening difficulties, so in cases with assumed biliary obstruction precise and early identification of cause and level of obstruction is vital to choose the suitable therapeutic management.

In our series, all our patients were complaining from yellowish discoloration of the skin and sclera as most common clinical complaint presented in all patients except for 6 cases. Our result agrees with Mathew et al. (2016) ${ }^{(14)}$.

In this study, MDCT identified the level of biliary obstruction in 69 patients with $100 \%$ accuracy which agreed with Mohamed et al. (2016) ${ }^{(15)}$.

Our study showed higher prevalence for male affection to biliary obstruction than females by $56.5 \%$ to $43.5 \%$, Rishi et al. (2015) ${ }^{(16)}$ showed different prevalence which is equal male to female affection.

Regarding most affected age group our study showed $60:<70$ years is the most affected group. While Rishi et al. (2015) ${ }^{(16)}$ stated that majority of his cases in the age group 41-60 years.

The results of our study showed degree of biliary duct dilatation initiated by malignant sources was more severe than that of benign sources similar to findings of Mohamed et al. (2016) ${ }^{(15)}$.

In our study It was found that calcular is main cause of benign OJ these were as those of Mathew et al. (2016) (14) who showed Calcular was responsible for $22 \%$ from causes of biliary obstruction.

Regarding nature of cause of biliary obstruction our study showed malignant dominance by $59.4 \%$ compared to $40.6 \%$ for
Zagazig University Medical Journals

benign which agrees with Narayanaswamy et al. (2015) ${ }^{(17)}$ which showed $66.7 \%$ for malignancy while 33.3 for benign causes,. But Mathew et al. (2016) ${ }^{(14)}$ stated benign causes were $56 \%$ while malignant was only $44 \%$.

Malignant stricture was identified by MDCT in 36 cases, 34 cases were confirmed by standard examination; a case had slight left sided intrahepatic dilatation with hyperenhancement of left intrahepatic duct wall and abrupt bile duct narrowing was identified as infiltrative cholangiocarcinoma. Nevertheless, ERCP identification was acute cholangitis. Second case was a well-known case of colon cancer on follow up a hepatic focal lesion was identified as a necrotic metastasis but cytology confirmed an abscess. Alternatively, a case of metastatic porta hepatis LN confirmed to be cholangiocarcinoma.

The SN, SP, PPV, NPV, and ACC of MDCT cholangiography in detection of malignant stricture were $94.4 \%$, 93.94, $94.44 \%, 93.93 \%$ and $94.2 \%$ respectively that come to an agreement with Mohamed et al. (2016) ${ }^{(15)}$ which results was $96.8 \%, 94.9$, $98.2 \%, 96.7 \%$ and $95.6 \%$.

Criteria for malignant stricture were irregular margins, invasion into neighboring tissues, abrupt termination of duct, longsegment involvement and enhancement in delayed or portal phases which was in agreement with criteria mentioned by Agrawal et al. (2018) ${ }^{(18)}$.

In cholangiocarcinoma 14 patients were included in our study responsible for $20.3 \%$ from total cases. MinIP images had extra value due to development in definition of stenosis in the district of the CBD bifurcation and precise evaluation of the extension of hilar cholangiocarcinoma.

Mathew et al. (2016) ${ }^{(14)}$ reported that cholangiocarcinoma $16 \%$ prevalence.

In pancreatic carcinoma in our series present in 14 patients responsible for $20.3 \%$ of from total cases, Mohamed et al. (2016) (15) in their study pancreatic carcinoma was responsible for $17.8 \%$ from the causes of biliary obstruction. Mathew et al. (2016) ${ }^{(14)}$ claimed that it affected $20 \%$ of cases. MDCT identified all the cases of pancreatic carcinoma with 100\% accuracy. Most studies 
agree the basis of diagnosing as ours: a hypodense mass relative to enhanced pancreatic tissue that may shows faint peripheral enhancement.

In Other malignancies there were 5 cases, 3 cases diagnosed as hepatic focal lesion, 1 case as GB carcinoma and gastric carcinoma, one case was wrongly diagnosed as metastatic focal lesion but proven as an abscess, Mohamed et al. (2016) ${ }^{(\mathbf{1 5})}$ results included 8 cases of intrahepatic malignant masses one case was diagnosed as benign stricture but pathologically proven to be metastasis from non-Hodgkin lymphoma. Tummala et al. (2013) ${ }^{(19)}$ reported extrinsic compression by focal lesion adjacent to bile duct in $3.2 \%$ of their patients (compared to $4.3 \%$ in our study).

Periampullary carcinoma was found in 3 cases, all were proven pathologically to be Periampullary carcinoma, it represented $4.3 \%$ of cases. Mohamed et al. (2016) ${ }^{(15)}$ showed higher affection rate (14\%) with 100 $\%$ accuracy that agreed with our results while Narayanaswamy et al. (2015) ${ }^{(17)}$ was $96 \%$.

Calcular was diagnosed in 17 cases, 1 case was diagnosed by MDCT as Calcular but proven to be a benign stricture (sludge) and another case which was diagnosed by MDCT as negative (no detected cause) proven to be a black cholesterol stone that was not visible. It was the most common cause of benign biliary obstruction in our study by contributing to $24.6 \%$ of the cases with SN 94.1\%, SP 98\%, PPV 94.1\%, NPV 98\% and ACC 97.1\%. Our results agrees with Rishi et al. (2015) ${ }^{(16)}$ that revealed in their study ACC of MDCT in detection of choledocholithiasis is $98 \%$ a, SN of $100 \%$ and SP of $97.4 \%$ showed also was the most frequently diagnosed cause of obstruction by $24 \%$. You et al. (2018) (20) mentioned intraductal high attenuating focal lesions were detected in enhanced or unenhanced scans subsequent the course of $\mathrm{CBD}$ as a criteria for diagnosis which agreed with our findings.

Four patients with post-operative iatrogenic bile duct injury were included which represented $5.8 \%$ from total causes, Heller et al. (2015) ${ }^{(21)}$ implies that iatrogenic is the second most common reason for benign biliary tract strictures after calcular which agreed with ours. Study showed free fluid in most cases which was noted in the abdomen accompanying fluid at GB bed. MDCT diagnosed all the patients correctly based on the history and findings making statistical measures for the diagnostic performance of MDCT in detecting bile duct injury as follow SN $100 \%$, SP $100 \%$, PPV 100\%, NPV $100 \%$ and ACC $100 \%$, El-gerby et al. (2006) ${ }^{(22)}$ and Meng et al. (2014) ${ }^{(23)}$ results showed same SN, SP, PPV, NPV \& ACC as ours for biliary leakage detection.

Four patients had porta hepatis lesions in our study. It contributed by $5.8 \%$ from causes which showed different results from Mohamed et al. (2016) ${ }^{(15)}$ was $4.4 \%$ only. Two cases were diagnosed as lymphoma involving porta-hepatis $\mathrm{LN}$ and other two cases as malignant metastatic porta-hepatis LN. Rishi et al. (2015) (16) stated that lymphoma represented $6 \%$ from causes of obstruction compared to $2.9 \%$ in our study and $100 \%$ ACC in detecting lymphoma which was the same finding as Mathew et al. (2016) (14). Our diagnostic performance was as follow SN 100\%, SP 98.48\%, PPV 75\%, NPV $100 \%$ and ACC $98.55 \%$.

Benign Stricture was diagnosed by MDCT in three cases, Two cases were proven to be due to recurrent cholangitis which agrees with Heller et al. (2015) ${ }^{(21)}$ that stated cholangitis considered one of the commonest causes of benign strictures. Mohamed et al. (2016) (15) mentioned MDCT criteria for identifying a benign stricture includes a smooth \& gradually elongated narrowing of CBD in a short section measuring less than one $\mathrm{cm}$ without presence of a mass. Our diagnostic performance for the detection of the benign strictures was as follow: SN 40\%,

SP $98.44 \%$, PPV $66.67 \%$, NPV 95.45\% and ACC 94.20\%. Mohamed et al. (2016) ${ }^{(15)}$ statistical results were SN $66.7 \%$, SP $80.2 \%$, PPV $80.2 \%$, NPV $91.7 \%$ and ACC $92.6 \%$ which was near to our results except for sensitivity as one case which was diagnosed by MDCT as benign stricture were proven by biopsy a small cholangiocarcinoma. While Mathew et al. (2016) (14) showed higher percentages as follows: SN 100\%, SP 97.8\%, PPV $83.3 \%$, NPV $100 \%$ and ACC $98 \%$. 
Inflammatory was diagnosed by MDCT in three cases, two cases were due to acute pancreatitis and one case was due to acute cholecystitis. Patel et al. (2013) (24) stated that acute cholecystitis diagnosis confirmed in the presence of GB wall thickening, GB distention peri-cholecystic fluid, inflammatory stranding \& subserosal oedema which agreed with our findings. Bonheur (2017) ${ }^{(25)}$ mentioned that biliary obstruction can occur from external compression of bile duct due to inflammation as pancreatitis or cholecystitis. Bollen (2016) (26), also mentioned that acute pancreatitis could lead to biliary complications including biliary obstruction.

Our study showed $100 \%$ SN, SP, PPV, NPV and ACC regarding the results of inflammatory causes. Mathew et al. (2016) ${ }^{(14)}$ study results presented that inflammatory cause of biliary obstruction has SN, SP, PPV, NPV and ACC of $100 \%$ which in turn agrees with our results.

A 13 years old female case with a cystic dilated segment involving extra hepatic biliary tree was diagnosed as congenital choledocal cyst type IA. Heller et al. (2015) ${ }^{(21)}$ mentioned modified Todani classification type IA represents cystic dilation of entire extrahepatic duct and also stated that type I is the most common type of choledocal cyst by $50-85 \%$. Our study showed $100 \%$ SN, SP, PPV, NPV and ACC to diagnosing the congenital cause of the biliary obstruction. Mathew et al. (2016) ${ }^{(14)}$ study results showed choledocal cyst has SN, SP, PPV, NPV and ACC of $100 \%$ which agrees with our results.

\section{CONCLUSION}

Due to MDCT low expense, easy access, shorter scan time and comparable diagnostic performance making it an excellent non-invasive substitute imaging modality, so we recommend using MDCT as an alternative to ERCP and MRCP for biliary imaging.

Conflict of Interest: Non declared.

Funding: No funding sources.

\section{REFERENCES}

(1) Chalya PL, Emmanuel SK, Mabula M. BMC research notes; 2011. Available at: http://www.biomedcentral.com/17560500/4/147.
(2) Lalani T, Couto CA, Rosen MP, Baker ME, Blake MA, Cash BD, et al. ACR appropriateness criteria on jaundice. American College of Radiology 2012.

(3) Kim HJ, Park DI, Park JH, Cho YK, Sohn CI, Jeon WK, et al. Multidetector computed tomography cholangiography with multiplanar reformation for the assessment of patients with biliary obstruction. Journal of Gastroenterology and Hepatology 2007; 22: 400-405.

(4) You M, Jung YY, Shin J. Role of magnetic resonance cholangiopancreatography in evaluation of choledocholithiasis in patients with suspected cholecystitis. J Korean Soc Radiol 2018; 78: 147-156.

(5) Zhang ZY, Wang D, Ni JM, Yu XR, Zhang $\mathbf{L}$, Wu WJ, et al. Comparison of threedimensional negative contrast CT cholangiopancreatography with threedimensional MR cholangiopancreatography for the diagnosis of obstructive biliary diseases. European Journal of Radiology 2011. Available at: http://dx.doi.org/10.1016/j.ejrad.2011.02.036.

(6) Fulcher AS. MRCP and ERCP in the diagnosis of common bile duct stones. Gastrointest Endosc 2002; 56: 178-182.

(7) Tongdee T, Amornvittayachan O, Tongdee R. Accuracy of multidetector computed tomography cholangiography in evaluation of cause of biliary tract obstruction. J Med Assoc Thai 2010; 93: 566-573.

(8) Tadros MY, Elia RZ. Current status of multidetector row helical CT in imaging of adult acquired pancreatic diseases and assessing surgical neoplastic respectability. Alexandria Journal of Medicine 2017; 53: 7-14.

(9) Tamm EP, Balachandran A, Bhosale P, Szklaruk J. Update on 3D and multiplanar MDCT in the assessment of biliary and pancreatic pathology. Abdom Imaging 2009; 34: 64-74.

(10) Ferrari FS, Fantozzi F, Tasciotti L, Vigni F, Scotto F, Frasci P. US, MRCP, CCT and ERCP: A comparative study in 131 patients with suspected biliary obstruction. Med Sci Monit 2005; 11: MT8-18.

(11) Richter GM. Pancreatic Adenocarcinoma: $\mathrm{CT}$ and PET/CT. Pancreatic Cancer. In: Neoptolemos J, Urrutia R, Abbruzzese J, Büchler M, eds. Pancreatic Cancer. Springer, New York, NY; 2018: 1-30.

(12) Yeh BM, Liu PS, Soto FA, Corvera CA, Hussain HK. MR imaging and CT of the biliary tract. RadioGrahics 2009; 29: 16691688. 


\section{Ahmed et al....}

(13) Boraschi B, Donati F. Biliary tract. In: Neri E, Caramella D, Bartolozzi C, eds. Image Processing in Radiology: Current Applications, Springer, first edition; 2008: 303-314.

(14) Mathew RP, Moorkath A, Basti RS, Suresh HB. Value and accuracy of multidetector computed tomography in obstructive jaundice. Pol J Radiol 2016; 81: 303-309.

(15) Mohamed HH, Koryem EM, El Olimy ASA, Abdelaal E. Accuracy of the multislice CT in diagnosis of biliary tract obstruction. The International Journal of Science and Technoledge 2016; 4: 228-237.

(16) Rishi M, Abdunnisar M, Suresh H. Value and accuracy of multidetector computed tomography in obstructive jaundice. Panacea Journal of Medical Science 2015; 5: 137-144.

(17) Narayanaswamy I, Erasu AR, Parkash HVR. The role of multidetector row computed tomography in biliary tract malignancy. Cancer Research Journal 2015; 3: 104-109.

(18) Agrawal P, Bo GJ, Bhattarai M, Shah SP, Agrawal M. Role of multidetector computed tomography in differentiating benign and malignant common bile duct strictures. West African Journal of Radiology 2018; 25: 21-27.

(19) Tummala P, Munigala S, Eloubeidi MA, Agarwal B. Patients with obstructive jaundice and biliary stricture \pm mass lesion on imaging prevalence of malignancy and potential role of EUS-FNA. J Clin Gastroenterol 2013; 47: 532537.

\section{Zagazig University Medical Journals}

(20) You M, Jung YY, Shin J: Role of magnetic resonance cholangiopancreatography in evaluation of choledocholithiasis in patients with suspected cholecystitis. J Korean Soc Radiol 2018; 78: 147-156.

(21) Heller MT, Borhani AA, Furlan A, Tublin ME. Biliary strictures and masses: An expanded differential diagnosis. Abdom Imaging 2015; 40: 1944-1960.

(22) El-gerby KM, Elsamak A, Khalil OE, Shawky KM, El-Kady H. Role of magnetic resonance imaging and magnetic resonance cholangiopancreatography versus helical computed tomography in evaluation of biliary obstruction. MD thesis, Faculty of Medicine, Zagazig University; 2006.

(23) Meng X, Huang W, Xie P, Chen X, Cai M, Shan $\mathbf{H}$, et al. Role of multi-detector computed tomography for biliary complications after liver transplantation. World J Gastroenterol 2014; 20: 11856-11864.

(24) Patel NB, Oto A, Thomas S. Multidetector CT of emergent biliary pathologic conditions. Radiographics 2013; 33: 1867-1888.

(25) Bonheur JL. Biliary obstruction. Medscape 2017: 1-5.

(26) Bollen TL. Imaging assessment of etiology and severity of acute pancreatitis. Pancreapedia 2016: $1-28$

How to cite this article: Ahmed MS, Hazim IT, Hamed AG, Ahmed MA.Role of Multidetector Computed Tomography in Biliary Obstruction.ZUMJ 2019; 25 (2); 207-215.DOI: 10.21608/ZUMJ.2019.6906.1008 